\title{
UTILIZAÇÃO DO BENCHMARKING NA GESTÃO ESTRATÉGICA DAS COOPERATIVAS AGROINDUSTRIAIS PARANAENSES
}

\section{EVALUATION OF BENCHMARKING AS A STRATEGIC MANAGEMENT TOOL FOR AGRICULTURAL COOPERATIVES}

\author{
MARCELO MACHOWSKI CAVALCANTI MARTINS \\ Professor da Faculdades do Brasil, UNIBRASIL, Brasil
Mestrando em Administração pela Pontifícia Universidade Católica do Paraná \\ Curitiba, PR - Brasil \\ ROBERTO MAX PROTIL \\ Professor da Pontifícia Universidade Católica do Paraná, PUC-PR, Brasil \\ ós-Doutorado pela Justus-Liebig-Universität Giessen \\ E-mail: roberto.proti@@ucpr.br \\ SERGIO LUIZ DOLIVEIRAS
Professor da Universidade Estadual do Centro-Oeste - UNICENTRO \\ Mestrado em Gestão Estratégica de Organizaçōes pela Universidade do Estado de Santa \\ Guarapuava, PR - Brasil \\ Guarapuava, PR - Brasil
E-mail: sldd@uol.com.br
}

\section{RESUMO}

Este artigo tem por objetivo caracterizar e avaliar o emprego do benchmarking no processo estratégico das cooperativas agroindustriais paranaenses que participaram do Programa de Revitalização das Cooperativas de Produção Agropecuária, RECOOP, no período compreendido entre 1998 e 2005. Trata-se de uma pesquisa exploratória que integrou pesquisa documental, entrevistas semi-estruturadas e observação direta para investigar o tema tendo como base principalmente as teorias de processos estratégicos sugeridas por Van De Ven (1992) e a descrição do processo de benchmarking formulada por Camp (1997). Utilizando diferentes técnicas e com apoio de um instrumento de análise desenvolvido para investigar as práticas de gestão estratégica convergentes com o benchmarking, foi possível concluir que o benchmarking é utilizado de uma forma ainda pouco estruturada pelas cooperativas paranaenses. Foram identificadas as práticas de gestão estratégica que podem representar uma fraqueza para a utilização do benchmarking pelas cooperativas, bem como as práticas que podem agir no sentido contrário, facilitando a adoção desta prática.

Palavras chave: Gestão Estratégica; Cooperativas Agroindustriais; Benchmarking.

\section{ABSTRACT}

This article deals with the characterization of benchmarking in the strategic process of agro-industrial cooperatives which participated of the Revitalization Program of Agricultural Cooperatives named Recoop, in the period between 1998 and 2005. This study had an exploratory character which incorporated documentary research, interviews and direct observation based on the theories of strategic processes suggested by Van De Ven (1992) and the benchmarking process formulated by Camp (1997). Using different techniques and with the support of an analytical tool developed to investigate the practices of strategic management, it was concluded that benchmarking is poorly used by the analyzed cooperatives. We identified management practices that may pose a strategic weakness for the use of benchmarking by the cooperatives, as well as practices that may act in the opposite direction, facilitating the adoption of this practice.

Key words: Strategic Management; Agricultural Cooperatives; Benchmarking. 


\section{INTRODUÇÃO}

0 atual ambiente de competição do qual participam as empresas tem dimensões globais e características constantemente alteradas por rápidas mudanças tecnológicas e de mercados. Neste cenário, pesquisadores e administradores vêm tentando criar e aperfeiçoar ferramentas que apóiem a formulação e implementação da estratégia empresarial. Entre estas ferramentas de gestão merece destaque o benchmarking.

Segundo Camp (1998) o benchmarking é a "busca das melhores práticas na indústria que conduzem ao desempenho superior". Para este autor, o benchmarking é um processo que começa com uma empresa identificando quais são suas forças e fraquezas em relação a empresas concorrentes e não concorrentes que possuem desempenho superior, ou seja, empresas que são capazes de melhor realizar processos necessários a um melhor desempenho. Este processo continua com a incorporação das melhores práticas das empresas que se adotou como referência, ou seja, com a adaptação e adoção de melhores formas de se realizar os processos necessários para um desempenho superior. Por fim, utilizando-se métricas que permitem mensurar as variações de desempenho e estabelecendo-se metas de desempenho a serem atingidas, as empresas passam a perseguir uma posição de superioridade em relação ao grupo de empresas tomado como referência. Em diversas oportunidades, podese observar que o benchmarking envolve uma série de processos e atividades, que são complexos, demandam também um enquadramento adequado e sistemático, conforme coloca DEROS et.all... (2006). Tanto no melhoramento de desempenho de processos logisticos, como nas atividades de pequenas e médias indústrias na cadeia de fornecedores de autopeças para grandes montadoras de automóveis, a utilização do benchmarking é intensivo.

A partir de formas distintas e com diferentes enfoques o benchmarking vem sendo aplicado por praticamente todos os tipos de organizações, tanto em indústrias como em empresas prestadoras de serviços. Assim como, nas iniciativas que envolvem as práticas ligadas a responsabilidade social empresaria (RSE), conforme aponta PRESLEY et. all.. (2010) auxiliando com um quadro de medição de desempenho, que pode ser utilizado em construções de prédios sustentaveis. O benchmarking também tem sido aplicado na agricultura, no segmento agroindustrial e por cooperativas. No entanto, a pesquisa científica nestes três últimos campos é particularmente escassa, especialmente no cenário brasileiro.

Em trabalhos recentes Barreiros \& Protil (2010), Protil et. al. (2009), Barreiros \& Protil (2008), Barreiros et. al. (2007), Peixe \& Protil (2007) verificaram que as cooperativas paranaenses, apesar da sua importância econômica e social, apresentam deficiências e ineficiências nas suas estratégias empresariais, que podem ser atribuído a utilização inadequada das ferramentas de gestão estratégica disponíveis, dentre as quais o benchmarking.

Em 1998 o governo federal institui o RECOOP, Programa de Revitalização de Cooperativas de Produção Agropecuária, com o objetivo de promover a melhoria da gestão das cooperativas agropecuárias, bem como, de promover uma adequação financeira de suas estruturas de débitos-passivos. Uma das exigências básicas 
deste programa era que as cooperativas promovessem uma profunda reengenharia organizacional dando um caráter mais profissional a sua gestão, o que indiretamente pressupunha a adoção de modernas ferramentas de gestão estratégica (Bialoskorski, 2010).

Diante destes fatos, depara-se com o seguinte problema de pesquisa:

- Qual o grau e a forma de utilização do "benchmarking” pelas cooperativas paranaenses, que optaram pelo RECOOP para a condução de suas estratégias organizacionais?

Portanto este artigo tem por objetivo caracterizar e discutir o processo de benchmarking no âmbito do processo estratégico das cooperativas agroindustriais paranaenses, ou seja, obter informações sobre o grau e forma de utilização do "benchmarking" pelas cooperativas paranaenses participantes do RECOOP para a condução de suas estratégias organizacionais.

0 artigo esta estruturado da seguinte forma: inicialmente apresenta-se a fundamentação teórica abordando questões conceituais sobre as organizações cooperativas, estratégia organizacional e processo estratégico, estratégia em cooperativas e benchmarking. Na seqüência apresenta-se a estratégia metodológica multi-métodos adotados neste trabalho para em seguida partir-se para a discussão dos resultados, onde são analisadas as diversas etapas do processo de benchmarking nas cooperativas estudadas. Por fim é apresentada uma conclusão com as limitações e sugestões para o aprofundamento deste estudo.

\section{FUNDAMENTAÇÃO TEÓRICA}

Segundo a OCEPAR (2010) as atuais 82 cooperativas agroindustriais do estado do Paraná com 129.590 associados são responsáveis por aproximadamente $20 \%$ do PIB do estado e $56 \%$ do PIB agrícola, além de empregarem mais de 48.484 funcionários. Estima-se que as cooperativas paranaenses são responsáveis direta ou indiretamente pela renda de aproximadamente um milhão de paranaenses, ou seja, 10\% da população do estado dependem de alguma forma das cooperativas. Apesar desta pujança econômica e importância social, estas organizações estão tendo dificuldades em definir estratégias empresariais mais eficientes em resposta às crescentes e complexas exigências do mercado nacional e internacional, perdendo, assim, competitividade frente a outras formas de organizações capitalistas. Para tentar compreender melhor este fenômeno e embasar a discussão sobre a utilização do benchmarking como importante ferramenta de gestão estratégica serão apresentados a seguir as bases teóricas-conceituais desta pesquisa.

\subsection{A ORGANIZAÇÃO COOPERATIVA}

De acordo com Hall (1984,p.23) as organizações podem ser assim definidas: 
“Uma organização é uma coletividade com uma fronteira relativamente identificável, uma ordem normativa, escalas de autoridade, sistemas de comunicação e sistemas de coordenação e afiliação; essa coletividade existe numa base relativamente contínua em um ambiente e se engaja em atividades que estão relacionadas, usualmente, com um conjunto de objetivos".

Para diversos autores, cooperativas são organizações muito particulares. Waack e Machado Filho (1999, p.149) caracterizam o sistema cooperativista agroalimentar da seguinte maneira:

O sistema cooperativista agroalimentar caracteriza-se pela associação de um grupo de (normalmente pequenos) produtores, em geral com uma base cultural comum, voltados para algumas atividades agrícolas específicas, que se reúnem sob um arcabouço organizacional e institucional próprio, a cooperativa singular;. Com algumas vantagens fiscais e doutrina própria, o modelo é amplamente utilizado na atividade agrícola ocidental. Com frequência, se re-agrupa nas chamadas cooperativas centrais (uma cooperativa de cooperativas), em um processo piramidal, voltado especialmente para ganhos de escala e de poder perante fornecedores e clientes.

Bialoskorski (2001) define as cooperativas como organizações intermediárias entre o mercado e os empreendimentos econômicos dos cooperados, sendo que sua principal missão é a de promover o incremento e integração dos empreendimentos de cada cooperado. Isso faz com que a existência das cooperativas dependa da prestação de serviços aos cooperados, dependência esta que não existe nas sociedades mercantis. Esta relação econômica diferenciada entre cooperativa e o cooperado é formalmente regulamentada pela legislação não como um ato comercial comum, mas como um ato cooperativo, e tem todas as suas características claramente descritas.

Para Waack e Machado Filho (1999) as características especiais das cooperativas tornam seu processo de gestão estratégica mais complexo do que o de outras organizações. Nesse sentido também as cooperativas, segundo Cook (2004), podem ser conceituadas como sendo de propriedade do usuário, devendo ser controlada por esse usuário e esse usuário é o principal beneficiário. Nesse sentido em diversas oportunidades os papeis se confundem no funcionamento da estrutura cooperativa proporcionando interesses contraditórios.

Aanálise das sociedades cooperativas sob a ótica da Nova Economia Institucional considera cinco problemas típicos dessas organizações, quais sejam, de horizonte, de incentivo, de portfólio, de controle e de influência, descritos a seguir (Cook, 1995; Zylbersztajn, 2003):

a) problema de horizonte: os cooperados tendem a rejeitar estratégias que impliquem imobilização de capital de longo prazo, devido à inalienabilidade das quotas partes.

b) problema de incentivo: a dificuldade de monitoramento das atividades dos cooperados pode gerar comportamento oportunista, na medida em que ocorra a concentração dos relacionamentos com a cooperativa somente nas situações de vantagens comerciais.

c) problema de portfólio: a dificuldade de capitalização de longo prazo gera o problema de portfólio, na medida em que a cooperativa passa a ter mais dificuldade de competição nos mercados de produtos com maior valor agregado, 
que exigem vultosos investimentos em tecnologia, marketing e distribuição;

d) problema de controle: nas cooperativas é comum a não separação entre a propriedade e o controle, o que pode ser eficiente em casos de processos de decisão simples e empresas pouco complexas, mas tende a constituir-se em fator limitante à eficiência da gestão dessas organizações, na medida do seu crescimento e do aumento de complexidade dos seus negócios.

e) problemas de influência: a acumulação das funções de propriedade e controle, dá margem ao surgimento de coalizões políticas entre os membros da cooperativa, na busca do exercício de cargos executivos, que são remunerados e providos de alta carga de poder.

Emerge dessas considerações, em que medida as ferramentas de gestão estratégica, e em especial o benchmarking poderia ser utilizado para solucionar ou pelo menos reduzir estes típicos problema das organizações cooperativas

\subsection{ESTRATÉGIA EM COOPERATIVAS}

Em um contexto contemporâneo, a estratégia pode ser entendida como resultante da prática organizacional. Esse campo de estudos recebe a denominação de estratégia como prática, do inglês, strategy-as-practice (SAP). Segundo essa perspectiva, a estratégia pode ser entendida como uma atividade dinâmica e situacional, altamente dependente do contexto de inserção organizacional, em constante elaboração e socialmente construída por meio das ações e interações dos múltiplos atores organizacionais (Jarzabkowski, 2005).

Segundo Whittington (1988), a SAP examina a estratégia sobre três ângulos distintos: (i) práticas, como conjuntos de tecnologias, rotinas, ferramentas, conceitos, idéias e procedimentos para pensar e agir utilizados pelos estrategistas; (ii) praxis, referente ao trabalho que é, de fato, executado ao se fazer estratégia, que, mesmo sendo um trabalho difuso, na medida em que envolve não apenas a alta administração, pode ser visto como acontecendo por meio de (sequiências de) episódios; e (iii) praticantes, isto é, os estrategistas, sejam eles proprietários, membros da alta administração ou funcionários (Whittington, 1988; 2002).

Por considerar-se a estratégia como o resultado de uma complexa combinação de respostas elaboradas por organizações aos desafios ambientais, torna-se interessante investigar se as condições particulares que caracterizam as cooperativas influenciam a montagem da estratégia nestas organizações.

Machado Filho et al. (2003) argumentam que no campo da estratégia o modelo cooperativo é de difícil gestão, em virtude da necessidade de atendimento de demandas muito heterogêneas, induzindo a um aumento natural do peso político no processo decisório. A governança se torna muito complexa, e grande parte do esforço gerencial se concentra nela. Além disso, reforçam que nas cooperativas brasileiras normalmente não há a separação entre propriedade e controle, na medida em que, 
geralmente, os gestores provêm do próprio quadro de associados, o que pode levar há dificuldades de gestão. Pela heterogeneidade dos interesses dos cooperados, as cooperativas agropecuárias ainda pulverizam seu esforço em negócios diversificados, com escalas de produção nem sempre suficientes, em detrimento da eficácia e da boa gestão.

Bialoskorski (2002) analisa as diferenças de estratégia que existem entre sociedades cooperativas e sociedades mercantis e postula que as cooperativas possuem duas "lógicas motoras": a social, preocupada com os cooperados associados, e a de mercado, enquanto que as sociedades mercantis possuem uma lógica única: a de mercado para a maximização dos lucros. Desta forma, o ambiente institucional das cooperativas impõe a estas demandas que não precisam ser consideradas pelas sociedades mercantis, as quais podendo orientar-se apenas pelas demandas de mercado, podem maximizar seus lucros.

Para Cook (2004) normalmente dentro do modelo cooperativista de agronegócios a perspectiva do produtor individual é de defesa. Há um estimulo em aumentar os retornos, por meio, da redução dos custos de produção e operação nos canais de comercialização e agregação de valor. Essas ações compensam os impactos econômicos do poder de mercado proporcionando a diminuição da probabilidade de perda nas rendas do produtor. Nessas oportunidades pode-se observar que os produtores, em geral, procuram as cooperativas para protegerem o valor atual e futuro de seus ativos agrícolas. Nesse sentido também a forma e a perspectiva de formação das cooperativas segue em um sentido de defesa dos produtores que se reúnem voluntariamente para buscar benefícios comuns.

Neste contexto a presente investigação busca compreender o processo de implantação da ferramenta benchmarking, enquanto prática estratégica. Ter o entendimento dessas práticas é fundamental para se perceber a própria vantagem competitiva desenvolvida pelas cooperativas. Isso denota uma contribuição pertinente, que poderá colaborar para o desenvolvimento dessa área de estudo, de modo a permitir a interpretação do próprio processo de gestão estratégica da organização, assim como sugerir alternativas de compreensão do processo de gestão de cooperativas agropecuárias.

\subsection{BENCHMARKING}

Simon e Von Der Gathen (2002) analisam 32 técnicas de planejamento e gerenciamento estratégico às quais chamam de instrumentos estratégicos e que podem ser classificados como instrumentos de análise ou conversão da estratégia, os quais podem ser utilizados no nível corporativo ou de unidade de negócio. Para estes autores, o benchmarking é um instrumento estratégico de análise aplicável no nível de unidade de negócio.

Apesar de ainda pouco estudado nos meios acadêmicos, o benchmarking vem sendo muito utilizado por empresas como uma ferramenta estratégica e diversas publicações não acadêmicas vêm apontando as vantagens de sua utilização por 
organizações de diferentes setores.

A falta de estudos científicos a respeito do tema é ressaltada por Yasin (2002). Este autor revisou a literatura publicada sobre benchmarking entre 1986 e 2002 e identificou 415 trabalhos que detalham a utilização desta ferramenta. Deste total, o autor analisou 159 trabalhos, concluindo que a literatura a respeito do benchmarking evoluiu de um enfoque sobre processos e atividades para, mais recentemente, abranger também a estratégia e os sistemas organizacionais. Verificou também que a maior parte dos trabalhos publicados é obra de practioners e que existe relativamente poucos trabalhos acadêmicos sobre o tema. Aplicações do benchmarking em cooperativas e na agricultura passaram a ser registradas em artigos científicos há pouco tempo.

Existem outras experiências como ressalta McKinnon (2009), por exemplo, dentro do setor de transportes no Reino Unido, por meio, de um programa patrocinado pelo Governo. Nessa situação o governo acabou por desenvolvendo uma pesquisa de benchmarking. A paritir dessa investigação passou-se a utilizá-la como parâmetro para o segmento de transporte. Também foi utilizada como uma ferramenta política para obter das empresas do setor privado uma ampliação da eficiência em suas operações, racionalizando a utilização dos recursos e diminuindo o impacto nas emissões de poluentes. Essa pesquisa foi realizada, no decorrer de 8 anos, nesse processo desenvolveu-se um modelo de benchmarking que serve de referencia em termos de eficiência e também forneceu informações vitais para a tomada de decisões do governo na área e auxiliou no desenvolvimento de campanhas públicas para promover uma maior eficiência dos transportes.

Em outro sentido Björklund (2010) aponta que o benchmarking pode auxiliar também para uma análise comparativa que pode ajudar, por sua vez, as empresas a aplicarem em suas iniciativas de Responsabilidade Social Empresarial (RSE). A autora relata um estudo no qual as o setor de compras passou a atuar em sintonia com os valores de RSE, fornecendo um modelo de práticas que auxiliam na melhoria do relacionamento tanto com fornecedores como com os clientes, proporcionando o desenvolvimento da área de compras dentro Organização. As iniciativas de RSE carecem de um método especifico e estruturado, o benchmarking fornece a oportunidade de auxiliar na criação de ferramentas e metodologias internas às empresas, que the forneçam condições de se desenvolverem. Esse estudo teve um caráter qualitativo e ilustrativo, buscando inspirar e orientar o desenvolvimento das organizações, respeitando as suas especificidades de atuação.

As implicações estratégicas do benchmarking para cooperativas agrícolas foram analisadas por Al-Hasan (2002) por meio de um estudo empírico envolvendo as duas maiores cooperativas do País de Gales, Reino Unido. Segundo o autor, a utilização do benchmarking poderia ser particularmente benéfica para as cooperativas, cujo gerenciamento e processo de tomada de decisão é reconhecidamente mais complicada do que de empresas "não-cooperadas", em função dos objetivos conflitantes dos shareolders que são também clientes.

Já Al-Hasan e Haines (2002) realizaram a análise do papel do benchmarking no incremento da performance de mercado, focando as cooperativas agrícolas. Seu estudo mostra que foi possível identificar as barreiras organizacionais para o serviço ao cliente, quais eram as áreas problema que contribuíam para a formação das barreiras e identificar a diferença de entre o que os gestores do negócio acreditam 
que os clientes querem receber enquanto serviços e o que os clientes realmente querem receber.

Programas de benchmarking aplicados à agricultura já existem e são praticados no Brasil. AFundação de Estudos Agrários “Luiz de Queiroz” (FEALQ), com pessoal ligado ao Departamento de Ciências Exatas da ESALQ/USP conduz, desde 2001, o Projeto BENTEVI - Benchmarking na Agricultura, que tem como objetivo "o levantamento de dados e a seleção de referenciais de excelência em indicadores de desempenho de processos relacionados à produção agrícola".

Para Camp (1998) o processo do benchmarking começa com o conhecimento sobre a própria empresa, baseada na avaliação de suas forças e fraquezas. Em seguida se faz a análise das forças e fraquezas dos concorrentes e líderes da indústria e se identificam quais padrões superiores devem ser seguidos, prossegue-se no processo incorporando as melhores práticas das empresas que se adotou como referência e, por fim, se estabelece um padrão de superioridade. O ciclo: conhecer sua operação, conhecer os líderes da indústria ou concorrentes, incorporar o melhor, conquistar a superioridade são descritos por Camp (1998, p. 2) como os "passos filosóficos básicos do benchmarking". O processo pode ser detalhado em um esquema com dez etapas, ou passos, mostrados na figura 1.

Figura 1 - Passos do Processo de Benchmarking

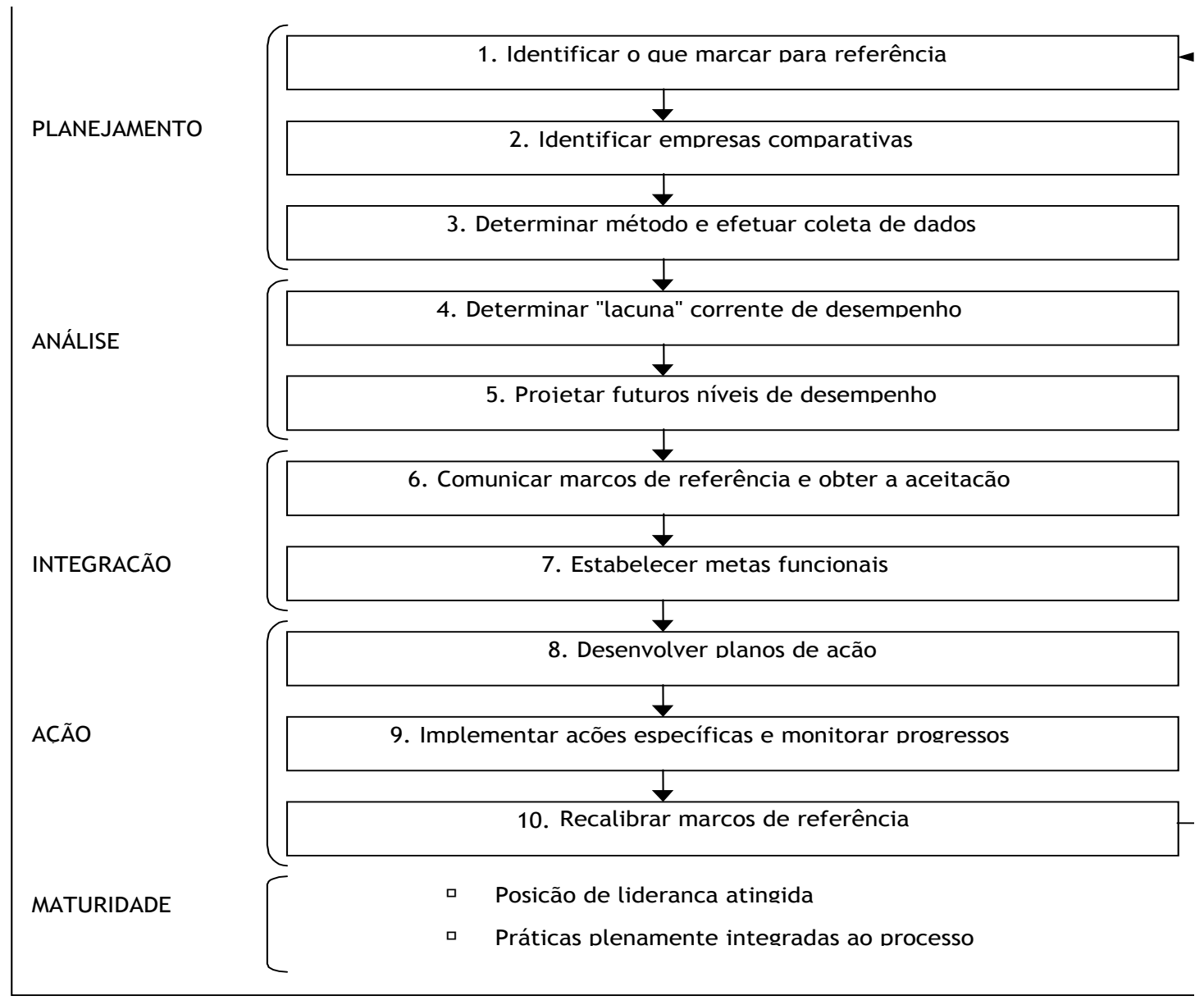

FONTE. CAMP (1998) 


\section{METODOLOGIA}

A pesquisa apresentada neste artigo teve um caráter exploratório cujo objetivo era investigar o emprego do benchmarking no processo estratégico das cooperativas agroindustriais paranaenses por meio de levantamento de dados secundários, entrevistas e análise de documentos. A teoria a ser utilizada como guia para coleta e análise de dados referente ao benchmarking foi o processo descrito por Camp (1998) e também os fatores críticos de sucesso deste processo apontados por Al-Hasan e Haines (2002), Camp (1997, 1998), Balm (1995) Fisher (2000) e Lincoln e Price (1997). Esta teoria permitirá também a generalização dos resultados para a teoria, seguindo os fundamentos da "generalização analítica" proposta por Yin (2005).

Já em relação ao processo estratégico a teoria utilizada foi constituída a partir do conjunto de recomendações de Van De Ven (1992) em relação à tipologia de processos estratégicos e aos conceitos de processo estratégico

Uma análise de documentos disponibilizados pelas cooperativas em seus sítios corporativos foi conduzida de forma a permitir a exploração das categorias de análise e de seus respectivos elementos de análise, os quais funcionam como indicadores de ocorrência dos fenômenos que se busca estudar melhor. A partir da complexidade da coleta e análise dos dados, foi necessário o uso de mais de uma estratégia de levantamento de informações, o que caracteriza o que Eisenhardt (1989) coloca como 'triangulação'.

Toda a parte de levantamento de dados foi feita em condições reais. Para analisar parte dos dados coletados um modelo foi construído para representar o emprego de práticas de gestão compatíveis com o processo de benchmarking nas cooperativas analisadas. A pesquisa transcorreu num ambiente de rotina real, não sendo modificada pelo pesquisador. Esta característica precisou ser reforçada em função deste ser um estudo ex post facto, no qual não existe o controle de variáveis pelo pesquisador e toda e qualquer influência sobre as variáveis deve ser evitada, conforme prescrevem Cooper e Schindler (2003).

Por fim, o nível de análise é o organizacional, e que as unidades de análise são as cooperativas agroindustriais do Estado do Paraná que participam do RECOOP. A escolha destas cooperativas como unidade de análise foi baseada na suposição de que a implantação do RECOOP representou um estímulo importante para a adoção de modernas práticas de gestão pelas cooperativas agroindustriais, entre as quais, possivelmente, o benchmarking.

Desta forma, não se adotou qualquer critério de seleção aleatória para a composição da amostra. Buscou-se de forma intencional e deliberada retirar do universo das cooperativas agroindústrias paranaenses aquelas que, no entender do pesquisador, tem um incentivo adicional para a adoção de modernas práticas de gestão.

Buscou-se entrevistar prioritariamente os presidentes das organizações, pois segundo vários autores entre os quais VAN DE VEN (1992), por se tratar de uma ferramenta de gestão estratégica, a efetiva adoção do Benchmarking depende não só 
do aval, mas também do envolvimento da presidência das empresas. Entrevistas com outros membros da alta-direção da empresa ou com executivos que participem do processo estratégico só foram realizadas em função da impossibilidade de entrevistar os presidentes ou por indicação destes.

Com relação a coleta de dados esta foi realizada em três etapas. Na primeira etapa foi feita uma pesquisa exploratória para investigar se as cooperativas agroindustriais paranaenses participantes do RECOOP demonstram ser organizações que possuem uma estratégia definida e que praticam benchmarking. Dados secundários foram levantados por meio de uma pesquisa documental nos web sites e relatórios anuais das cooperativas. Na segunda etapa foram realizadas entrevistas semi-estruturadas por telefone junto aos presidentes das cooperativas ou, na impossibilidade do presidente, com executivos que participem do processo estratégico da organização. Um questionário foi utilizado pelo pesquisador como apoio para as entrevistas que teve, como objetivo declarado, conhecer melhor as práticas de gestão estratégica das cooperativas agroindustriais paranaenses. Os objetivos não declarados são dois:

1. Identificar o emprego de práticas de gestão compatíveis com as etapas do processo de benchmarking no processo estratégico das cooperativas, bem como ligadas aos seus fatores críticos de sucesso, segundo a percepção da alta administração das cooperativas.

2. Identificar práticas de gestão que viabilizam a formulação, implementação e controle da estratégia utilizada pelas cooperativas agroindústrias paranaenses que participam do RECOOP.

Com relação ao questionário foram feitas 19 afirmativas que descrevem as práticas de gestão estratégica convergentes com o processo de benchmarking, cobrindo todas as fases do processo e também aos fatores críticos de sucesso para a condução bem-sucedida do benchmarking.

O questionário continha ainda cinco questionamentos referentes ao ferramental estratégico que poderia estar sendo empregado pelas cooperativas, sendo que uma das ferramentas sobre a qual os executivos precisavam manifestar-se era o próprio benchmarking.

Foram conduzidas entrevistas semi-estruturadas por telefone com os presidentes das cooperativas, os quais foram interrogados sobre se discordam ou concordam com que as atividades que caracterizam cada um dos elementos de análise são realizadas em suas organizações. Foram perguntados também se estas atividades são documentadas e comunicadas formalmente e se há reuniões periódicas de posicionamento para a alta direção sobre o processo e se esta emite instruções e posicionamentos a respeito aos funcionários.

O quadro 1 oferece uma visão sintética das perguntas de pesquisa, categorias de análise e elementos utilizados no presente trabalho.

Os posicionamentos possíveis variavam ao longo de uma escala de cinco pontos, com os seguintes pontos: Discordo Fortemente (1), Discordo (2), Neutro (3), Concordo (4), Concordo Fortemente (5). Existe ainda a possibilidade de uma sexta resposta, que não compõe a escala, e que permite ao entrevistado não responder. 
“Não sei/Não quero responder (0)".

Quadro 1 - Perguntas de Pesquisa, Categorias e Elementos de Análise

\begin{tabular}{|c|c|c|}
\hline PERGUNTAS DE PESQUISA & $\begin{array}{l}\text { CATEGORIA DE } \\
\text { ANÁLISE }\end{array}$ & ELEMENTOS DE ANÁLISE \\
\hline $\begin{array}{l}\text { As cooperativas agroindustriais } \\
\text { paranaenses participantes } \\
\text { do RECOOP demonstram ser } \\
\text { organizações que possuem uma } \\
\text { estratégia definida e que praticam } \\
\text { benchmarking? }\end{array}$ & $\begin{array}{c}\text { RECOOP, } \\
\text { estratégia e } \\
\text { benchmarking }\end{array}$ & $\begin{array}{l}\text { - Participação no RECOOP. } \\
\text { - Emprego de planejamento estratégico. } \\
\text { - Utilização do benchmarking. } \\
\text { - Definição de missão, visão ou valores. }\end{array}$ \\
\hline $\begin{array}{l}\text { É possível identificar o emprego } \\
\text { de práticas de gestão que } \\
\text { correspondam a etapas do processo } \\
\text { de benchmarking no processo } \\
\text { estratégico das cooperativas? }\end{array}$ & $\begin{array}{c}\text { Processo de } \\
\text { benchmarking. }\end{array}$ & $\begin{array}{l}\text { - Grau de compatibilidade das práticas } \\
\text { com: } \\
\text { - a etapa "Planejamento", } \\
\text { - a etapa "Análise", } \\
\text { - a etapa "Integração", } \\
\text { - a etapa "Ação", } \\
\text { - o estágio de "Maturidade". } \\
\text { - Apoio da alta administração e } \\
\text { formalização. }\end{array}$ \\
\hline $\begin{array}{l}\text { As cooperativas agroindustriais } \\
\text { paranaenses que participam do } \\
\text { RECOOP dispõe de ferramentas de } \\
\text { gestão estratégica para viabilizar } \\
\text { a formulação, implementação e } \\
\text { controle da estratégia? }\end{array}$ & $\begin{array}{l}\text { Ferramental } \\
\text { estratégico }\end{array}$ & $\begin{array}{l}\text { - Declaração de: } \\
\text { - emprego de planejamento } \\
\text { - estratégico, } \\
\text { - emprego de balanced scorecard, } \\
\text { - emprego de sistema de informação } \\
\text { gerencial informatizado, } \\
\text { - } \quad \text { emprego de benchmarking, } \\
\text { - } \quad \text { participação no RECOOP. }\end{array}$ \\
\hline $\begin{array}{l}\text { Como se caracteriza o emprego } \\
\text { do benchmarking no processo } \\
\text { estratégico de uma das cooperativas } \\
\text { agroindustriais paranaenses que } \\
\text { participam do RECOOP no período } \\
\text { compreendido entre } 1998 \text { e } 2005 ?\end{array}$ & $\begin{array}{l}\text { Benchmarking } \\
\text { no processo } \\
\text { estratégico }\end{array}$ & $\begin{array}{l}\text { - Descrição da estrutura e } \\
\text { características de documentos } \\
\text { estratégicos da cooperativa. } \\
\text { - Descrição do processo estratégico } \\
\text { da cooperativa por meio da análise } \\
\text { longitudinal de documentos e ações } \\
\text { estratégicas deliberadas. } \\
\text { - Descrição das ações estratégicas } \\
\text { deliberadas em que o emprego de } \\
\text { benchmarking tenha sido identificado } \\
\text { por meio do uso do termo benchmarking } \\
\text { ou por referências que permitam } \\
\text { caracterizar etapas do processo de } \\
\text { benchmarking. } \\
\text { - Descrição de eventos em que } \\
\text { identificou-se o emprego de } \\
\text { benchmarking por meio de entrevistas } \\
\text { e observação direta, mesmo que o } \\
\text { termo benchmarking não tenha sido } \\
\text { empregado. }\end{array}$ \\
\hline
\end{tabular}




\section{DISCUSSÃO DOS RESULTADOS}

\subsection{RECOOP, ESTRATÉGIA E BENCHMARKING}

A primeira fase da pesquisa procurou responder as seguintes questões referentes às cooperativas analisadas: "Participa do RECOOP?", "Possui Planejamento Estratégico?”, “Pratica Benchmarking?”.

Para tanto, dados secundários foram procurados nos sites das cooperativas, mas apenas cinco entre as 26 cooperativas que mantêm sites na internet disponibilizaram seus relatórios anuais de forma on-line. Além disso, não foram identificados sites de 11 cooperativas. Dois e-mails foram enviados ao endereço eletrônico de contato informado nos sites das cooperativas que não divulgaram seus relatórios on-line, mas nenhuma resposta foi obtida. Por isso, foi solicitado o apoio da OCEPAR para consultar os relatórios anuais das cooperativas. Ao final, foram lidos os relatórios anuais de 25 cooperativas.

Para a questão “Possui Planejamento Estratégico?” foram encontradas sete ocasiões de emprego do termo "planejamento estratégico", onze situações que indicam a possibilidade de emprego desta ferramenta e, por fim, para 19 cooperativas, não foi possível identificar nenhum indício de emprego do planejamento estratégico.

Em relação à questão "Pratica Benchmarking?", nesta etapa da pesquisa não foi possível identificar nenhuma referência direta ao emprego desta ferramenta. Foram encontradas onze possíveis situações de emprego.

A indicação mais forte de possível emprego foi identificada na CASTROLANDA, em função da adoção do modelo de gestão da FNQ por esta cooperativa (CASTROLANDA; 2005). O processo de gestão da FNQ em seu tópico "Gestão baseada em processos e informações", prescreve o emprego de processos que correspondem ao processo de benchmarking.

A FNQ é a fundação Nacional da Qualidade, trata-se de uma entidade privada criada em 1991 e que tem por objetivo promover a Excelência na Gestão e a melhoria da competitividade das organizações do Brasil (FNQ, 2010). Trata-se de uma empresa sem fins lucrativos e que promove concursos como o Prêmio Nacional da Qualidade, apresentando um ranking das empresas que se destacam, por meio, da utilização de ferramentas e práticas ligadas a Programas de Qualidade em seus processos de produção e gestão. respondido). O RECOOP teve uma frequência de quatro ocorrências, com duas citações diretas.

\subsection{PROCESSO DE BENCHMARKING}

Numa segunda fase da pesquisa foram realizadas entrevistas com a alta 
administração das cooperativas de forma a investigar o grau em que, na percepção destes executivos, ocorre o emprego de práticas de gestão estratégicas compatíveis com as etapas do processo de benchmarking, bem como o grau em que atendem aos fatores críticos de sucesso do benchmarking. Também se investigou o emprego de algumas ferramentas administrativas que permitem a definição, implantação e monitoramento estratégico.

Um questionário com afirmativas que descrevem as práticas de gestão estratégica convergentes com o processo de benchmarking foi elaborado, cobrindo todas as fases do processo e também as práticas necessárias para a condução bemsucedida do benchmarking. O questionário continha ainda cinco questionamentos referentes ao ferramental estratégico que poderia estar sendo empregado pelas cooperativas, sendo que uma das ferramentas sobre a qual os executivos precisavam manifestar-se era o próprio benchmarking.

De um universo de 29 cooperativas, obtiveram-se nove entrevistas, que representam $31,03 \%$ do universo pesquisado. Se forem consideradas as 37 cooperativas originalmente pesquisadas, as cooperativas cujos dados foram analisados somariam $24,32 \%$ do universo analisado.

Apenas dois presidentes responderam pessoalmente ao pesquisador. As demais informações foram obtidas junto a dois diretores, um gerente geral e a quatro gerentes. Cinco das nove entrevistas realizadas foram obtidas por telefone e quatro foram realizadas por formulários enviados por correio.

Para verificar em que medida práticas de gestão convergentes com os passos do processo de benchmarking estariam presentes no processo estratégico das cooperativas, os administradores estratégicos das cooperativas foram convidados a se posicionar em relação a uma série de afirmativas que descreviam as práticas de gestão compatíveis com o benchmarking. Os posicionamentos possíveis variavam ao longo de uma escala de 5 pontos: Discordo Fortemente (1), Discordo (2), Neutro (3), Concordo (4), Concordo Fortemente (5). Existe ainda a possibilidade de uma sexta resposta, que não compõe a escala, e que permite ao entrevistado não responder. “Não sei/Não quero responder (0)".

Quanto mais próxima a mediana obtida estiver do valor 5, máximo valor arbitrado para a escala, maior seria o grau de concordância em relação à presença daquela prática de gestão entre as cooperativas analisadas. Em seguida, outra análise foi feita, reunindo as questões em blocos que correspondem a todos os passos de uma determinada etapa do processo de benchmarking.

Por fim, para cada cooperativa foram elaboradas duas medianas, uma reunindo as médias dos posicionamentos para as 19 afirmativas relativas às etapas do processo de benchmarking e outra para as medianas das afirmativas relativas aos fatores críticos de sucesso do processo de benchmarking. Um gráfico foi então construído, o qual permite apresentar a posição de cada cooperativa em termos de sua posição relativa à convergência para o processo de benchmarking.

A figura 2 apresenta o posicionamento dos gestores estratégicos em relação a cada uma das 19 afirmativas relativas às práticas de gestão convergente com o processo de benchmarking. Cada uma das cinco possíveis respostas da escala Likert 
para cada afirmativa estão expressos em \%.

Figura 2 - Posicionamentos para as Práticas de Gestão Convergentes com o Processo de Benchmarking

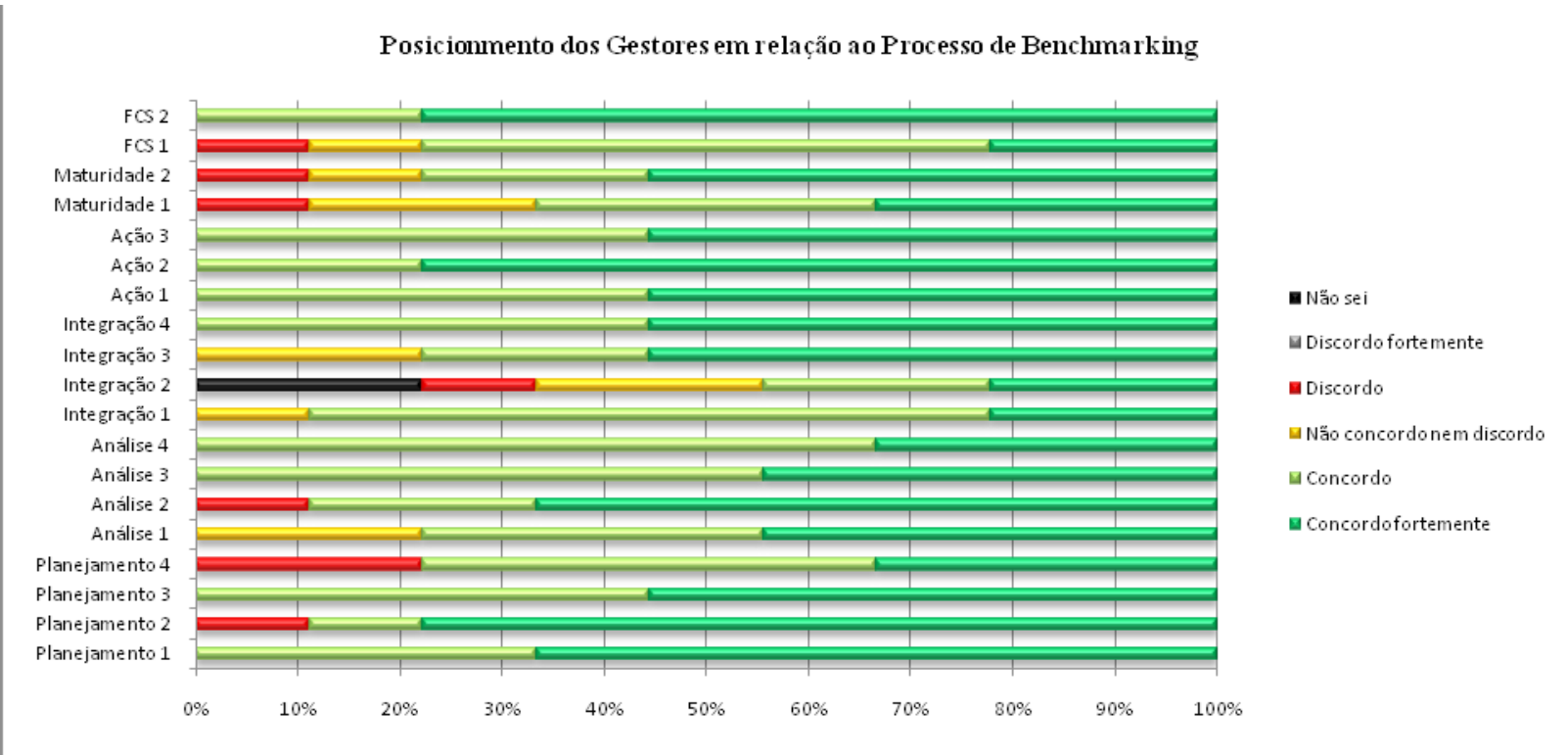

Este gráfico mostra que as práticas de gestão necessárias para a condução do processo de benchmarking pelas cooperativas, segundo o processo prescrito por CAMP (1998), estão presentes, segundo a percepção dos gestores estratégicos das cooperativas, em diferentes graus de intensidade.

Com base nos estudos de CAMP (1997) verificou-se, que valores abaixo de quatro são indicativos de que práticas de gestão convergentes com o processo de benchmarking estão presentes de forma tênue ou insuficiente, indicando nestes casos que um bom processo de benchmarking seria mais difícil de ser desenvolvido.

Desta forma, tomando o conjunto das cooperativas analisadas como referência existem sete práticas de gestão estratégica que precisariam estar presentes em intensidade maior nas cooperativas analisadas de tal forma que estas organizações possam realizar um processo de benchmarking mais consistente com o que prevêem as referências teóricas adotadas: Planejamento 2 (mediana 3,5), Planejamento 4 (mediana 3,5), Análise 2 (mediana 3,5), Integração 2 (mediana 3,5), Maturidade 1 (mediana 3,5), Maturidade 2 (mediana 3,5). Além disso, FCS 1 (mediana 3,5), também precisaria ser reforçado.

\subsection{FASE DE PLANEJAMENTO}

O quadro abaixo demonstra como variaram os posicionamentos ao longo do continuum discordo fortemente - concordo fortemente para as práticas estratégicas convergentes com a etapa Planejamento do processo de benchmarking. Para tanto utilizou-se as medianas obtidas para estas manifestações. 
Quanto mais próxima de cinco for a mediana, maior a convergência daquela prática com o processo de benchmarking.

Quadro 1 - Posicionamento em relação às etapas da fase de planejamento

\begin{tabular}{|l|c|c|c|c|c|c|c|}
\cline { 2 - 9 } \multicolumn{1}{c|}{} & \multicolumn{7}{c|}{ Escala Likert } \\
\hline \multicolumn{1}{|c|}{ ETAPAS DA FASE DE PLANEJAMENTO } & Mediana & 0 & 1 & 2 & 3 & 4 & 5 \\
\hline $\begin{array}{l}\text { Planejamento 1: A cooperativa costuma comparar } \\
\text { suas unidades umas com as outras ou comparar-se a } \\
\text { outras cooperativas, agroindústrias não cooperativas } \\
\text { ou organizações que ela considere boas empresas }\end{array}$ & 4,5 & $0 \%$ & $0 \%$ & $0 \%$ & $0 \%$ & $33 \%$ & $67 \%$ \\
\hline $\begin{array}{l}\text { Planejamento 2; É feito um planejamento prévio } \\
\text { para decidir que produtos, processos e práticas com- } \\
\text { parar a outras empresas }\end{array}$ & 3,5 & $0 \%$ & $0 \%$ & $0 \%$ & $11 \%$ & $78 \%$ \\
\hline $\begin{array}{l}\text { Planejamento 3: A empresa faz um planejamento } \\
\text { prévio para escolher a que empresas se comparar }\end{array}$ & 4,5 & $0 \%$ & $0 \%$ & $0 \%$ & $0 \%$ & $44 \%$ & $56 \%$ \\
\hline $\begin{array}{l}\text { Planejamento 4: É feito um planejamento prévio } \\
\text { para coletar as informações que serão comparadas }\end{array}$ & 3,5 & $0 \%$ & $0 \%$ & $0 \%$ & $44 \%$ & $33 \%$ \\
\hline
\end{tabular}

Constata-se que práticas de gestão estratégicas ligadas ao processo decisório e ao planejamento da coleta dos dados necessários ao benchmarking representam claramente o "calcanhar de Aquiles" da fase de "Planejamento".

As eventuais lacunas nas condições necessárias para um efetivo planejamento do benchmarking poderiam ser preenchidas por uma série de fatores que ainda precisam ser investigadas. Talvez estes fatores incluam maior conhecimento sobre o processo de benchmarking, informações gerenciais de melhor qualidade, identificação precisa de pontos fortes e fracos, ameaças e oportunidades, recursos humanos capacitados, recursos financeiros ou condições políticas.

\subsection{FASE DE ANÁLISE}

O quadro 2 apresenta de forma consolidada as práticas de gestão convergentes com as etapas da fase de "Análise" do processo de benchmarking. As práticas que tiveram a menor mediana na etapa de Análise foram as sintetizada pela afirmativas Análise 1 e Análise 2.

Este resultado sugere que as cooperativas estariam interessadas, ou teriam condições apenas, de aplicar o processo de benchmarking de forma mais superficial e que práticas estratégicas ligadas à análise detalhada dos processos, produtos e práticas próprios e daqueles tomados como referência são seus pontos fracos. 
Quadro 2 - Posicionamento em relação às etapas da fase de análise

\begin{tabular}{|l|c|c|c|c|c|c|c|}
\cline { 2 - 8 } \multicolumn{1}{c|}{} & \multicolumn{7}{c|}{ Escala Likert } \\
\hline \multicolumn{1}{c|}{ ETAPAS DA FASE DE ANÁLISE } & Mediana & 0 & 1 & 2 & 3 & 4 & 5 \\
\hline $\begin{array}{l}\text { Análise 1: A cooperativa realiza uma análise para } \\
\text { compreender em detalhes os próprios produtos, } \\
\text { processos e práticas, bem como os das empresas às } \\
\text { quais quer comparar-se. }\end{array}$ & 4 & $0 \%$ & $0 \%$ & $0 \%$ & $22 \%$ & $33 \%$ & $44 \%$ \\
\hline $\begin{array}{l}\text { Análise 2: Nesta análise a cooperativa estabelece } \\
\text { a diferença de desempenho atual em relação ao } \\
\text { grupo de referência, verificando se está acima, } \\
\text { abaixo ou no mesmo nível das organizações a que } \\
\text { se compara }\end{array}$ & 3,5 & $0 \%$ & $0 \%$ & $11 \%$ & $0 \%$ & $22 \%$ & $67 \%$ \\
\hline $\begin{array}{l}\text { Análise 3: A cooperativa examina como as práticas } \\
\text { das empresas líderes podem ser incorporadas aos } \\
\text { seus próprios processos }\end{array}$ & 4,5 & $0 \%$ & $0 \%$ & $0 \%$ & $0 \%$ & $56 \%$ & $44 \%$ \\
\hline $\begin{array}{l}\text { Análise 4: A cooperativa consegue projetar, identi- } \\
\text { ficar, imaginar quais níveis de desempenho precisa } \\
\text { alcançar no futuro para ser melhor que as organiza- } \\
\text { ções a que se comparou }\end{array}$ & 4,5 & $0 \%$ & $0 \%$ & $0 \%$ & $0 \%$ & $67 \%$ & $33 \%$ \\
\hline
\end{tabular}

\subsection{FASE DE INTEGRAÇÃO}

A seguir apresenta-se o quadro 3 consolidando as medianas para as práticas convergentes com as etapas da fase de "Integração" do processo de benchmarking.

\section{Quadro 3 - Posicionamento em relação às etapas da fase de integração}

\begin{tabular}{|c|c|c|c|c|c|c|c|}
\hline \multirow[b]{2}{*}{ ETAPAS DA FASE DE INTEGRAÇÃO } & \multicolumn{7}{|c|}{ Escala Likert } \\
\hline & Mediana & 0 & 1 & 2 & 3 & 4 & 5 \\
\hline $\begin{array}{l}\text { Integração 1: A cooperativa comunica as desco- } \\
\text { bertas decorrentes da análise das empresas } \\
\text { líderes a toda a organização, obtendo aceitação } \\
\text { e compreensão }\end{array}$ & 4 & $0 \%$ & $0 \%$ & $0 \%$ & $11 \%$ & $67 \%$ & $22 \%$ \\
\hline $\begin{array}{l}\text { Integração 2: As descobertas decorrentes da aná- } \\
\text { lise das empresas líderes são transformadas em } \\
\text { declaração de princípios que passam a orientar } \\
\text { operações da cooperativa }\end{array}$ & 3,5 & $22 \%$ & $0 \%$ & $11 \%$ & $22 \%$ & $22 \%$ & $22 \%$ \\
\hline $\begin{array}{l}\text { Integração 3: A cooperativa estabelece as metas } \\
\text { operacionais da mudança e as práticas que pre- } \\
\text { cisam ser absorvidas para ser, no futuro, melhor } \\
\text { que as organizações a que se comparou }\end{array}$ & 4 & $0 \%$ & $0 \%$ & $0 \%$ & $22 \%$ & $22 \%$ & $56 \%$ \\
\hline $\begin{array}{l}\text { Integração 4: A cooperativa assegura que as } \\
\text { metas e práticas que precisam ser adotadas para } \\
\text { garantir uma posição de liderança sejam consid- } \\
\text { eradas nos planejamentos formais da organização } \\
\text { por meio de planos de ação }\end{array}$ & 4,5 & $0 \%$ & $0 \%$ & $0 \%$ & $0 \%$ & $44 \%$ & $56 \%$ \\
\hline
\end{tabular}

A primeira prática convergente com a etapa Integração do processo de 
benchmarking, Integração 1, aparece com mediana 4,0 considerada suficientemente baixa para comprometer um eventual processo de benchmarking. Pode-se supor que falhas na comunicação das descobertas decorrentes da análise das empresas líderes a toda a organização prejudicam o processo de benchmarking. É interessante notar que esta prática é também analisada como um fator crítico de sucesso do processo de benchmarking.

A prática Integração 2 obteve uma mediana 3,5, a mais baixa de todas as registradas, o que faz com que um eventual processo de benchmarking esteja bastante comprometido. Pode-se supor que as cooperativas não compreendem a importância da transformação das descobertas decorrentes da análise das empresas líderes em declaração de princípios que passam a orientar operações da cooperativa.

Com relação à Integração 3, esta se refere a um importante aspecto do benchmarking, a definição do que precisa ser alterado na organização para que esta seja melhor que as organizações a que se comparou. Esta prática tem uma semelhança muito grande com relação à Integração 4, que lida com o mesmo assunto. A diferença entre as duas proposições é sutil. Enquanto Integração 3 fala de estabelecer metas operacionais de mudança e práticas que precisam ser adotadas para garantir uma posição de liderança em relação às organizações a que se comparou, a afirmativa Integração 4 fala em assegurar que metas e práticas que precisam ser adotadas para garantir uma posição de liderança sejam consideradas nos planejamentos formais via planos de ação.

No entanto, a afirmativa Integração 4 obteve uma mediana de 4,5. Conforme CAMP (1997) o fato de a Integração 4 parecer mais próxima de outras práticas estratégicas, como o planejamento estratégico, ajuda na formulação da hipótese de que a utilização de ferramentas estratégicas facilita o emprego do benchmarking.

\subsection{FASE DE AÇÃO}

O quadro abaixo consolida as informações para a fase de Ação do processo de benchmarking.

Quadro 4 - Posicionamento em relação às etapas da fase de ação

\begin{tabular}{|l|c|c|c|c|c|c|c|}
\cline { 2 - 8 } \multicolumn{1}{c|}{} & \multicolumn{7}{|c|}{ Escala Likert } \\
\hline \multicolumn{1}{c|}{ ETAPAS DA FASE DE AÇÃO } & Mediana & 0 & 1 & 2 & 3 & 4 & 5 \\
\hline $\begin{array}{l}\text { Ação 1: São implementados planos para atingir estas } \\
\text { metas de desempenho futuro }\end{array}$ & 4,5 & $0 \%$ & $0 \%$ & $0 \%$ & $0 \%$ & $44 \%$ & $56 \%$ \\
\hline $\begin{array}{l}\text { Ação 2: Os progressos obtidos com estas ações são moni- } \\
\text { torados periodicamente }\end{array}$ & 4,5 & $0 \%$ & $0 \%$ & $0 \%$ & $0 \%$ & $22 \%$ & $78 \%$ \\
\hline $\begin{array}{l}\text { Ação 3: As metas, bem como os produtos, processos } \\
\text { e práticas adotados como referência, são revisados, } \\
\text { ajustados ou substituídos em função do monitoramento, } \\
\text { caso isso seja necessário }\end{array}$ & 4,5 & $0 \%$ & $0 \%$ & $0 \%$ & $0 \%$ & $44 \%$ & $56 \%$ \\
\hline
\end{tabular}

As três afirmativas criadas para medir as práticas de gestão convergentes com 
as etapas da fase de Ação apresentaram medianas elevadas, o que indica que poucos problemas poderiam surgir na implantação de um processo de benchmarking em relação a esta etapa. Em função deste resultado pode-se inferir que as cooperativas já adotam práticas de gestão estratégica mais convergentes com as que descrevem etapas da fase de ação do processo de benchmarking, o que facilitaria a implementação e a gestão do processo de benchmarking nestas organizações.

\subsection{FASE DE MATURIDADE}

A fase de maturidade é aquela atingida pela empresa após desenvolver o processo de benchmarking. Nessa fase a empresa atingiu uma posição de liderança em relação às organizações a que se comparou e já incorporou as melhores práticas que as organizações de referência poderiam oferecer. As afirmativas que permitem verificar práticas convergentes com uma posição de maturidade são apresentadas no Quadro 5.

Quadro 5 - Percepção em relação às etapas da fase de maturidade

\begin{tabular}{|l|c|c|c|c|c|c|c|}
\cline { 2 - 8 } \multicolumn{1}{c|}{} & \multicolumn{7}{c|}{ Escala Likert } \\
\hline \multicolumn{1}{|c|}{ ETAPAS DA FASE DE INTEGRAÇÃO } & Mediana & 0 & 1 & 2 & 3 & 4 & 5 \\
\hline $\begin{array}{l}\text { Maturidade 1: Usando estas práticas de gestão } \\
\text { a cooperativa atingiu a posição de liderança em } \\
\text { relação às organizações a que se comparou }\end{array}$ & 3,5 & $0 \%$ & $0 \%$ & $11 \%$ & $22 \%$ & $33 \%$ & $33 \%$ \\
\hline $\begin{array}{l}\text { Maturidade 2: As melhores práticas das organiza- } \\
\text { ç̃̃es às quais a cooperativa comparou-se foram } \\
\text { integradas pela cooperativa }\end{array}$ & 3,5 & $0 \%$ & $0 \%$ & $11 \%$ & $11 \%$ & $22 \%$ & $56 \%$ \\
\hline
\end{tabular}

Com relação a esta fase verifica-se, que as cooperativas analisadas estão longe de possuir um ambiente que descreva a fase de maturidade em termos de benchmarking. Estas baixas medinas podem estar associadas a problemas de gestão nas etapas anteriores do processo de benchmarking, que fazem com que as cooperativas tenham dificuldade em atingir a etapa de Maturidade deste processo e pela falta de percepção a respeito do verdadeiro sentido do processo de benchmarking, que faz com que as cooperativas não atinjam uma posição de maturidade satisfatória neste processo.

\subsection{FATORES CRÍTICOS DE SUCESSO (FCS) DO BENCHMARKING}

As práticas referentes aos Fatores Críticos de Sucesso (FCS) não fazem parte do processo de benchmarking, mas constituem condições necessárias para que este seja bem-sucedido. O quadro abaixo mostra que de maneira geral as cooperativas analisadas possuem dificuldade em estruturar o registro e comunicação do processo de benchmarking, fatores fundamentais para o sucesso desta pratica gerencial. 


\section{Quadro 6 - Média para os Fatores Críticos de Sucesso}

\begin{tabular}{|l|c|c|c|c|c|c|c|}
\cline { 2 - 7 } \multicolumn{1}{c|}{} & \multicolumn{5}{c|}{ Escala Likert } \\
\hline \multicolumn{1}{c|}{ ETAPAS DA FASE DE ANÁLISE } & Mediana & 0 & 1 & 2 & 3 & 4 & 5 \\
\hline $\begin{array}{l}\text { FCH1: A utilização destas práticas de gestão se dá } \\
\text { de forma estruturada, em um processo de registro } \\
\text { e comunicação formal. }\end{array}$ & 3,5 & $0 \%$ & $0 \%$ & $11 \%$ & $11 \%$ & $56 \%$ & $22 \%$ \\
\hline $\begin{array}{l}\text { FCH2; A utilização destas práticas de gestão tem o } \\
\text { apoio e envolvimento explícitos da alta direção da } \\
\text { cooperativa }\end{array}$ & 4,5 & $0 \%$ & $0 \%$ & $0 \%$ & $0 \%$ & $22 \%$ & $78 \%$ \\
\hline
\end{tabular}

A partir dessas constatações pode-se inferir que o apoio da alta-direção a práticas de gestão convergentes com as do processo de benchmarking facilita a implementação deste processo nas cooperativas e que a falta de práticas ligadas à comunicação do processo representa uma ameaça para a implementação bem sucedida do benchmarking nas cooperativas.

\subsection{CONVERGÊNCIA PARA BENCHMARKING}

Por fim, uma última análise baseada em Van De Vem (1993) permite demonstrar graficamente a posição de cada cooperativa em termos de sua convergência para o processo de benchmarking em função das práticas de gestão analisadas. Para isso foram elaboradas duas médias para cada cooperativa:

- Mediana dos posicionamentos para as 19 afirmativas relativas às etapas do processo de benchmarking,

- Mediana das afirmativas relativas aos fatores críticos de sucesso do processo de benchmarking.

Essas medianas permitem que um gráfico seja construído para demonstrar a posição de convergência de benchmarking para cada uma das cooperativas, de forma a comparar a posição relativa de cada uma em relação à outra. Estas medianas são apresentadas no quadro 7.

\section{Quadro 7 - Medianas - convergência de Benchmarking}

\begin{tabular}{|c|c|c|}
\hline COOPERATIVA & MÉDIANA ETAPAS & $\begin{array}{c}\text { MEDIANA FATORES } \\
\text { CRITICOS DE SUCESSO (FCS) }\end{array}$ \\
\hline Cooperativa 1 & 4,5 & 4,5 \\
\hline Cooperativa 2 & 5 & 4 \\
\hline Cooperativa 3 & 5 & 5 \\
\hline Cooperativa 4 & 4 & 3 \\
\hline Cooperativa 5 & 3,5 & 3,5 \\
\hline Cooperativa 6 & 5 & 4,5 \\
\hline Cooperativa 7 & 3,5 & 4 \\
\hline Cooperativa 8 & 4 & 4 \\
\hline Cooperativa 9 & 4,5 & 4 \\
\hline
\end{tabular}


A figura 3 apresenta o gráfico de convergência para benchmarking, o qual foi dividido em quatro quadrantes, formados por dois eixos que representam as medianas para os eixos " $x$ " e $\ddot{y}$ ”. No gráfico são assinaladas com um círculo azul as posições ocupadas pelas cooperativas 1, 2, 3, 6 e 9, que são as que, em seu conjunto, demonstraram possuir as práticas de gestão mais convergentes com o processo de benchmarking, bem como melhor atender aos fatores críticos de sucesso necessários à implementação do benchmarking.

Figura 3 - Posição relativa em termos de convergência para Benchmarking

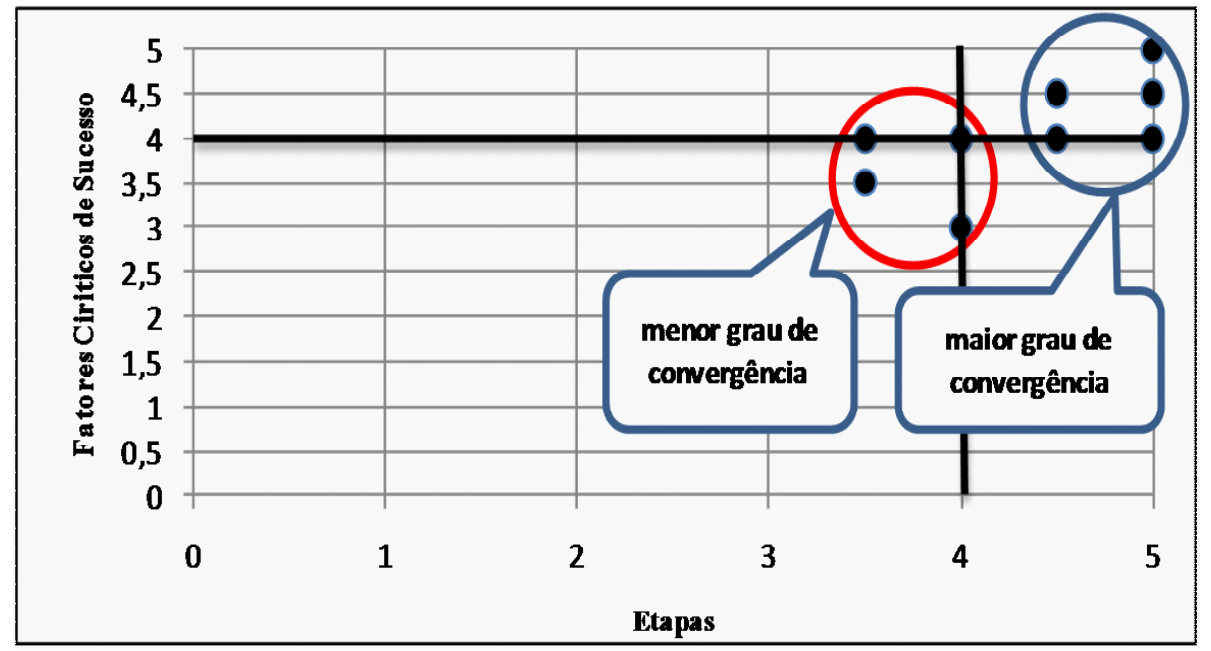

Um segundo grupo representado pelo circulo vermelho esta posicionado à esquerda do eixo que define a mediana obtida para a mensuração das etapas do processo estratégico (eixo $\mathrm{x}$ ), e gravitando acima e abaixo da mediana para as práticas ligadas aos FCS (eixo y). Neste grupo estão as cooperativas 4,5,7 e 8.

Este gráfico demonstrando as posições relativas das cooperativas em termos de convergência de benchmarking poderia ser utilizado para um benchmarking de práticas de gestão convergente com o processo de benchmarking entre as cooperativas. As cooperativas que quisessem aprimorar suas práticas de gestão convergentes com o processo de benchmarking poderiam propor que as cooperativas 1,2,3,6 e 9 fossem suas organizações de referência e poderiam absorver práticas de gestão delas.

\section{CONCLUSÃO}

Este trabalho demonstrou que o termo benchmarking não é desconhecido das cooperativas e isso torna possível que pesquisas explicativas e causais sejam iniciadas para aprofundar o conhecimento deste tema em relação a este universo organizacional e à teoria do benchmarking.

Os documentos analisados pelo pesquisador não permitem afirmar que as cooperativas agroindustriais paranaenses participantes do RECOOP praticam 
o benchmarking e, em poucos casos foi possível identificar a estratégia destas organizações.

Não obstante, durante a segunda fase da pesquisa, com o emprego de uma ferramenta de diagnóstico, foi possível identificar como variavam o emprego das práticas de gestão que correspondam a etapas do processo de benchmarking no processo estratégico das cooperativas, o que revelou que práticas estavam mais presentes, quais, ao contrário, estavam representadas de forma mais tênue.

Da mesma forma, foi possível constatar que as cooperativas agroindustriais paranaenses que participam do RECOOP dispõem, pelo menos assim o afirmam os seus executivos, de ferramentas de gestão estratégica para viabilizar a formulação, implementação e controle da estratégia, entre estas, o benchmarking.

Para isso concorreram os resultados das três fases da pesquisa, mas, mente, a ferramenta de diagnóstico utilizado para investigar as práticas de gestão estratégica convergentes com o benchmarking, a qual transformou os passos filosóficos do benchmarking em categorias de conceitos que podem ser distinguidos de outros processos estruturados e mensurados como entidades fixas (variáveis), cujos atributos podem variar ao longo de escalas numéricas.

Finalizando, sugere-se que as cooperativas participantes do RECOOP utilizem meios de comunicação internos e externos para divulgar elementos que demonstrem que possuem uma estratégia estabelecida. 0 esforço de definição destes elementos e sua divulgação poderão contribuir para que a estratégia organizacional seja adequadamente formulada e implementada, contribuindo para uma melhor percepção a respeito da capacidade das cooperativas em sobreviver e prosperar, tanto por parte de observadores externos como por parte dos cooperados.

Trata-se de um trabalho inicial, em função disso existem limitações. A investigação junto às Cooperativas Agroindustriais buscou refletir as suas especificidades, entretanto, fica claro que há um amplo campo de pesquisas a ser aprofundado, no qual, apenas foram realizados os primeiros passos. As ferramentas de Estratégias são variadas e podem ser muito melhor utilizadas nesse importante segmento do Estado do Paraná. Sugere-se que sejam realizados futuros estudos que busquem investigar de maneira mais detalhada as necessidades e o desenvolvimento de ferramentas e de Estratégias nas Cooperativas deste setor produtivo.

A pesquisa contribuiu levantando que existe há carências de Gestão em uma parte significativa das Cooperativas de Agronegócios do Paraná, em relação a utilização da ferramenta "benchmarking"e demonstrou a necessidade de uma melhor profissionalização do corpo diretivo e dos colaboradores dessas entidades.

Houve limitações em relação ao número de adesões a pesquisa, apesar de não afetar o seu grau de significância, aponta para a necessidade de futuras sondagens que possam indicar as transformações na Gestão das Cooperativas. Isso auxiliara no processo de compreensão do processo de tomada de decisões Estratégicas, bem como na utilização de ferramentas de Gestão como o Benchmarking. 


\section{REFERÊNCIAS}

AL-HASAN, S. The strategic implications of benchmarking for agricultural co-operatives and farmer controlled enterprises in Walles. In: THOMAS, B; AL-HASAN, S; SPARKES, A. Innovation and knowledege transfer in the Welsch agri-food industry. Welsch Enterprise Institute Reader. University of Glamorgan, p. 61 - 82, 2002.

AL-HASAN, S.; HAINES, M. The role of benchmarking in the improvement of marketing performance: an aplication to the welsch co-operative sector. In: THOMAS, B; ALHASAN, S; SPARKES, A. Innovation and knowledege transfer in the Welsch agri-food industry. Welsch Enterprise Institute Reader. University of Glamorgan, p. 83 - 110, 2002.

BALM, G. J. Benchmarking: um guia para o profissional tornar-se - e continuar sendo - o melhor dos melhores. Rio de Janeiro: Qualymark, 1995.

BARREIROS, R. F., PROTIL, R. M. Proposta de um Mapa Estratégico para Cooperativas Agroindustriais - A Importância da Perspectiva "Relação com Cooperados". Anais do XXXIV ENANPAD, Rio de Janeiro, 2010.

BARREIROS, R. F., PROTIL, R. M., MOREIRA, V. R. Fatores de Influência do Processo Decisório em Nível Estratégico nas Cooperativas Agropecuárias do Paraná. Informe Gepec, Toledo, v. 22, n. 2, p. 30-45, 2007.

BARREIROS, R. F., PROTIL, R. M., MOREIRA, V. R. Processo Decisório nas Cooperativas Agroindustriais do Paraná: Uma Análise Comparativa Utilizando o Modelo Racional e o Modelo Político de Decisão. RCO - Revista de Contabilidade e Organizações, Ribeirão Preto, v. 2, n. 4, p. 3-22, 2008.

BIALOSKORSKI NETO, S. Agronegócio cooperativo. In: BATALHA, M. O. Gestão Agroindustrial, São Paulo: Atlas, 2001, capítulo 12, p 628-655.

BIALOSKORSKI NETO, S. Estratégias e cooperativas agropecuárias: um ensaio analítico. In: SEMINÁRIO DE POLÍTICA ECONÔMICA EM COOPERATIVISMO E AGRONEGÓCIOS DA UFV, 2002, [S.L.].

BIALOSKORSKI NETO, S. $1^{\circ}$ Workshop Internacional de Tendencia do Cooperativismo. http://www.fearp.usp.br/ sigbial/inserir_out2002/I\%20WITC.pdf acessado em 12 de Novembro de 2010.

BJÖRKLUND, M. Benchmarking tool for improved corporate social responsibility in purchasing. Benchmarking: An International Journal, (pp. 340 - 362) - Volume 17 issue 3 Special Issue: Benchmarking the Greening of Business, Published: 2010

CAMP, R. C. Adaptar criativamente. HSM Management. São Paulo, ano 1, 3, p. 64 68, jul./ago. 1997.

CAMP, R. C. Benchmarking: identificando, analisando e adaptando as melhores práticas que levam à maximização da performance empresarial: o caminho da qualidade total. 3. ed. SãoPaulo: Pioneira, 1998. 
CASTROLANDA. Site corporativo. Disponivel em http://www.castrolanda.com.br. Acesso em em 21. nov. 2005.

COOK, M. L. The Future of US Agricultural Cooperatives: A Neo Institutional Approach. American Journal of Agricultural Economics, n. 77, 1153-1159, 1995.

COOK, M. CHADDAD,F. R. Redesigning cooperative boundaries: the emergence of new models. American Journal of Agricultural Economics Vol. 86, Iss. 5; pg. 1249, 2004.

COOPER, D.R; SCHINDLER, P. S. Métodos de pesquisa em administração. 7. ed. Porto Alegre: Bookman, 2003.

DEROS, B.M. YUSOF, S.M., SALLEH, A.M. A benchmarking implementation framework for automotive manufacturing SMEs. Benchmarking: An International Journal, (pp. 396 - 430) - Volume 13 issue 4 , Published: 2006.

EISENHARDT, K. Building theories from case study research. The Academy of Management Review, Briarcliff Manor, v.14, p. 532-550, oct., 1989.

ESALQ/USP - ESCOLA SUPERIOR DE AGRICULTURA LUIZ DE QUEIROZ/ UNIVERSIDADE DE SÃO PAULO. O que é o projeto BENTEVI? Disponível em: http://bentevi.esalq.usp. br/ Acesso em 21 fev 2005.

FISHER, J, G. Benchmarking para otimizar o desempenho.2. reimp. São Paulo: Clio Editora, 200?.

FNQ. Critérios de Excelência 2005: o estado da arte da gestão para a excelência do desempenho e o aumento da competitividade. 2. tir. São Paulo: FNQ, 2005, acesso em 15nov.2010.

HALL, R. H. Organizações: estrutura e processos. 3. ed. Rio de Janeiro: Prentice Hall do Brasil, 1984.

JARZABKOWSKI, P. Strategy as practice: an activity-based aproach. Thousand Oaks: Sage, 2005.

LINCOLN, S.; PRICE, A. O que os livros de benchmarking não dizem. HSM Management. São Paulo, ano 1, 3, p. 70 - 74, jul./ago. 1997.

MACHADO FILHO, C. A. P.; MARINO, M.K.;CONEJERO, M.A . Gestão estratégica em cooperativas agroindustriais. Disponível em <http://www.fia.com.br/PENSA/>, 2003.

MCKINNON, Alan. Benchmarking road freight transport: Review of a governmentsponsored programme. Benchmarking: An International Journal, (pp. 640 - 656) - Volume 16 issue 6 Special Issue: Benchmarking of supply chain perform, 2009.

MINTZBERG, Henry; WATERS, James. Of strategies, deliberate and emergent. In: MCKIERNAN, Peter. Historical evolution of strategic management. I. Brookfield, Dartmouth Publishing Company, 1996.

OCEPAR - Organização e Sindicato das Cooperativas do Estado do Parana. www. 
ocepar.org.br, acessado em 5/11/2010.

PEIXE, J. B., PROTIL, R. M. Eficiência Econômica e Social das Cooperativas Agroindustriais Paranaenses: Proposta de um Modelo de Avaliação. Informe Gepec, Toledo, v. 22, n. 2, p. 2-15, 2007.

PRESLEY, A., MEADE, L. Benchmarking for sustainability: an application to the sustainable construction industry. Benchmarking: Benchmarking: An International Journal, (pp. 435 - 451) - Volume 17 issue 3 Special Issue: Benchmarking the Greening of Business, 2010

PROTIL, R. M., FERNANDES, A. C., SOUZA, A. B. K. Avaliação da Pesquisa Agropecuária em Cooperativas Agroindustriais utilizando um Modelo de Scorecard Dinâmico. RCO - Revista de Contabilidade e Organizações, Ribeirão Preto, v. 3, n. 5, p. 62-79, 2009.

RUMELT, Richard P.. Evaluation of strategy: theory and models. In: SCHENDEL, Dan; HOFER, Charles W. Strategic management: a new view of ness policy ans planning. Boston, Little, Brown and Company, 1979.

SIMON, H; von der GATHEN. A. Das grosse Handbuch der Strategieinstrumente. Frankfurt/New York: Campus Verlag, 2002.

VAN DE VEN, A. H. Suggestions for studying strategic process: a research note. Strategic Managment Journal. v. 13, Special Issue: Strategy Process: Managing Corporate SelfRenewal, p. 169 - 191, [S.L.] Summer, 1992

WAACK, R.S.; MACHADO FILHO C. P. M. Adminsitração estratégica em cooperativas agroindustriais. WORKSHOPBRASILEIRODEGESTÃODESISTEMASAGROALIMENTARES, 2, 1999, Ribeirão Preto.

WHITTINGTON, R. Environmental, Structure and Theories of Strategic Choice. Journal of Management studies, v. 25(6): 521-536, 1988.

WHITTINGTON, R. O que é estratégia. São Paulo: Pioneira Thomson Learning, 2002.

YASIN, M. M. The theory and practice of benchmarking: then and now. Benchmarking: an international journal, Bradford, v. 9, 3, p. 217 - 243, 2002.

YIN, R. K. Estudo de caso: planejamento e métodos. 3. ed. Porto Alegre:

Bookman, 2005. 


\section{DADOS DOS AUTORES:}

\section{Marcelo Machowski Cavalcanti Martins}

Pontifícia Universidade Católica do Paraná

Rua Imaculada Conceição, 1155 - Prado Velho

Curitiba, PR - Brasil

$80215-901$

\section{Roberto Max Protil}

Pontifícia Universidade Católica do Paraná

Rua Imaculada Conceição, 1155 - Prado Velho

Curitiba, PR - Brasil

$80215-901$

\section{Sergio Luiz Doliveira}

Universidade Estadual do Centro Oeste - UNICENTRO

Rua Presidente Zacarias, 875 - Campus Guarapuava

Guarapuava, PR - Brasil

$85015-430$ 